\title{
SENECA: a New Program for the Analysis of Single-Electron Devices
}

\author{
L. R. C. FONSECA ${ }^{\mathrm{a}}$, A. N. KOROTKOV ${ }^{\mathrm{b}}$ and K. K. LIKHAREV ${ }^{\mathrm{b}}$ \\ ${ }^{\mathrm{a} D e p a r t m e n t ~ o f ~ A p p l i e d ~ M a t h e m a t i c s ~ a n d ~ S t a t i s t i c s, ~ S t a t e ~ U n i v e r s i t y ~ o f ~ N e w ~ Y o r k, ~ S t o n y ~ B r o o k, ~ N Y ~ 11794-3600, ~}$ \\ ${ }^{6}$ Department of Physics, State University of New York, Stony Brook, NY 11794-3800
}

\begin{abstract}
We describe a new and efficient method for numerical study of the dynamics and statistics of single-electron systems presenting arbitrary combinations of small tunnel junctions, capacitances, and voltage sources. The method is based on the numerical solution of a master equation describing the time evolution of the probabilities of the electric charge states of the system, with iterative refining of the operational set of states. The method is able to describe very small deviations from the "classical" behavior of a system, due to finite speed of applied signals, thermal activation, and macroscopic quantum tunneling of charge (cotunneling). As an illustration, we briefly study the leakage rate in single-electron traps and the accuracy of several devices (turnstile, pump, and a hybrid circuit) suitable as standards of dc current.
\end{abstract}

Keywords: Quantum Electronics, Devices, Simulation, Coulomb Blockade Tunneling, Nanotechnology

\section{INTRODUCTION}

The manipulation of single-electrons in nanoscale circuits has opened the way to a new family of electronic devices [1]. These devices consist of one or several small conducting electrodes ("islands"), separated from each other and from external electrodes by tunnel junctions. As a result of the very small capacitance of the islands and junctions, if an extra electron tunnels into (out of) an island, the electrostatic potential of the island may change considerably, affecting the tunneling of the following electrons. Such correlated tunneling makes it possible to control the motion of single-electrons/holes in the circuit by the application of suitable potentials to external electrodes. Several applications of this effect have been proposed [1], including logic switchs, memory cells, ultrasensitive electrometers, and current standards.
A major problem in the design of single-electron circuits is an accurate determination of the probability of errors. Such errors (e.g. a misplacement of a singleelectron in the circuit), may be due to several factors, including thermal activation, finite speed of externally applied signals, and macroscopic quantum tunneling of charge (cotunneling) [1]. For a circuit of any reasonable complexity, these factors are intractable analytically.

The purpose of this report is to describe SENECA [3] (standing for Single-Electron NanoElectronic Circuit Analyzer), a new computer algorithm designed for studies of the dynamics and statistics of singleelectron devices, including order-of-magnitude calculation of rare errors due to all phenomena listed above. SENECA can handle single-electron circuits consisting of an arbitrary combination of lumped components (excluding resistors at this stage of 
development), at arbitrary temperature and time dependence of externally applied signals.

\section{THE ALGORITHM}

Tunneling events in single-electron circuits may occur on very different time scales determined by the corresponding tunneling rates, as predicted by theory [2]. This is why it is impossible to use Monte Carlo simulation if it is necessary to take into account rare tunneling events (say, when determining errors) on the background of much more frequent events. An alternative method is to calculate the probabilities of all possible states of the system (each characterized by a particular charge configuration) solving a master equation [2]. If the tunnel resistances are much larger than the quantum of resistance $R_{Q}=25.6 \mathrm{k} \Omega$, the master equation is a linear set of markovian equations

(a)
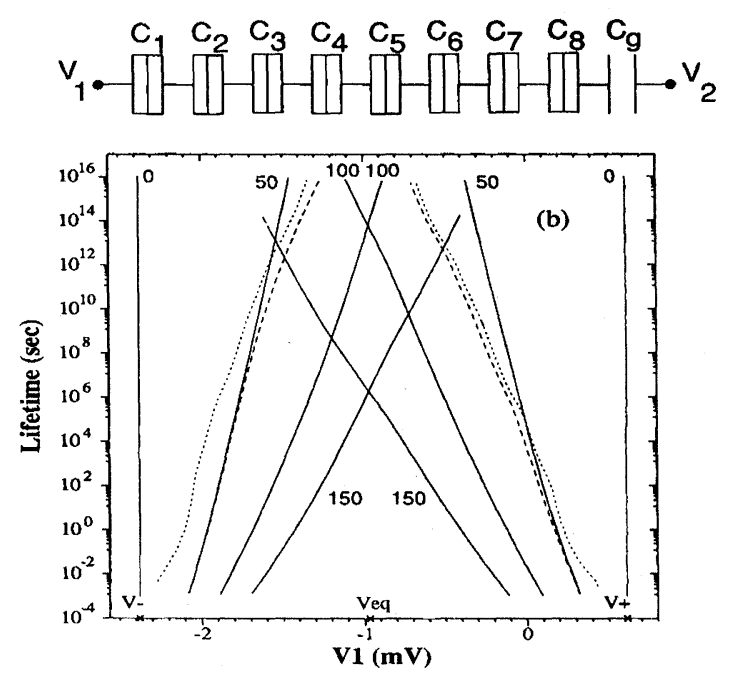

FIGURE 1 (a) The 8-junction single-electron trap. Tunnel capacitances and resistances are $\mathrm{C}_{\mathrm{t}} \approx 0.2 f F$ and $\mathrm{R}_{\mathrm{t}} \approx 1 \mathrm{M} \Omega$; (b) Lifetimes of the static states of the 8-junction trap for different temperatures. The curves with negative (positive) slope describe the lifetime of the empty (full) cell. Solid lines: classic approximation for $T=0$ (vertical lines), 50, 100, and $150 \mathrm{mK}$. Dotted lines: cotunneling included, $T=0$. Dashed lines: cotunneling included, $50 \mathrm{mK}$. At $100 \mathrm{mK}$ and $150 \mathrm{mK}$, the results including cotunneling virtually coincide with the classic ones describing the time evolution of the probabilities of the states of the system.

The necessity to store information about all possible charge states of the system (and the tunneling rates between them) imposes a major limitation on our approach. However, this limitation can be rectified to a large extent by taking into account the hierarchy of probabilities, where only states with probabilities higher than a certain threshold are considered at a time. In the program, this hierarchy is used in an iterative procedure, each iteration corresponding to a passage along the total time interval, which is define by the physical problem in study. For iteration number $\mathrm{n}$ we define a certain threshold probability $P_{t h}(n)$ as the lower bound for the probabilities of the states considered in that particular iteration. A natural choice of $P_{t h}(n)$ is such that each new iteration accounts roughly to one more order of cotunneling. The program stops if some deviation from the classic behavior of the system is found, or if the minimum threshold probability of interest is reached.

\section{EXAMPLES}

In order to demonstrate the capabilities of SENECA, we have analyzed a version of a simple SEL circuit, the so-called "single-electron trap" [2]. It consists of an array of tunnel junctions directly connected to one external electrode at one end and to a second electrode via a capacitor at another end (Fig 1(a)). The array presents an energy barrier for tunneling electrons due to electrostatic charging of the islands. The barrier may be suppressed by the application of a voltage bias between the external electrodes exceeding some threshold voltage $V_{+}$. In this case, a singleelectron is injected into the trap (state 1); its removal (yielding state 0 ) requires the applied voltage to go below another threshold $V_{-}<V_{+}$. In the region $V_{-}<V$ $<V_{+}$the circuit is bistable: the additional electron may be either in or out of the trap, depending on its history.

Even within the bistable loop $\left[V_{-}, V_{+}\right]$, thermal fluctuations and cotunneling may result in spontaneous switchings $0 \rightarrow 1$ and $1 \rightarrow 0$. Our goal was to use 
SENECA to find the rates of these switching events. The results of such calculation are shown in Figure 1(b). At relatively high temperatures $(\sim 100 \mathrm{mK}$ and above), the results of calculations with and without account of cotunneling virtually coincide, because the main mechanism of spontaneous switching at those temperatures is the classical thermally-activated hopping over the energy barrier. At lower temperatures, cotunneling gradually becomes the main mechanism of spontaneous switching.

Looking at the absolute numbers, we see that the rate of spontaneous switching in the 8-junction trap may be extremely low: lifetimes in excess of $10^{16}$ sec (i.e. $\tau \geq$ $\left.10^{26} \mathrm{C} / \mathrm{G}\right)$ are possible at achievable temperatures $(\sim 50$ $\mathrm{mK})$ within a relatively broad parameter window aroung the equilibrium voltage $V=V_{e q}$ (at $V_{e q}$ the energies of the empty and full cell are the same).

Our second problem was calculating and comparing the accuracy of several possible standards of dc current, namely the turnstile, the pump, and a hybrid

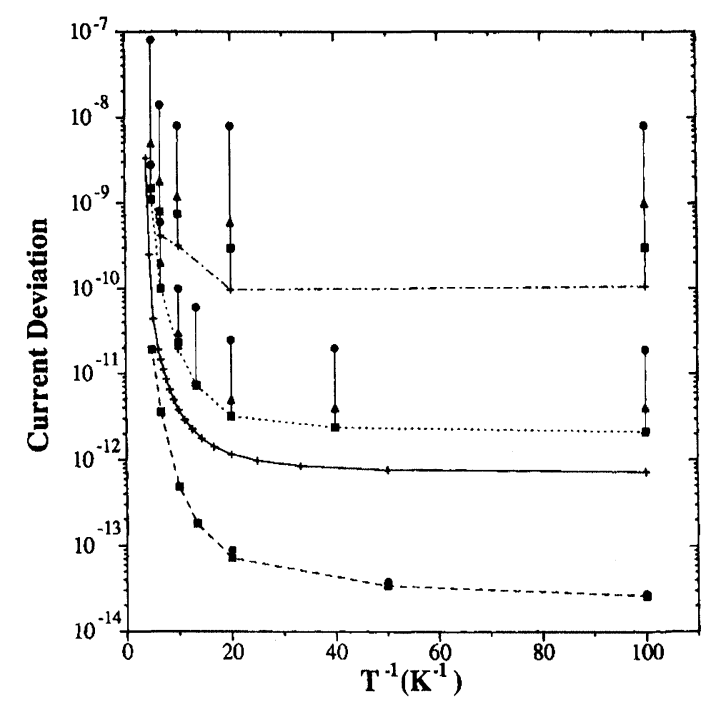

FIGURE 2 Current deviation in non-biased (solid line) and biased 5 -junction pump (dashed line), biased 6-junction h-pump (dotted line), and 8-junction turnstile (dash-dotted line) at $f=10 \mathrm{MHz}$ as a function of temperature. The ends of the vertical bars show the maximum current deviation if the inflection point is missed (in biased devices) by $5 \%$ (box), $10 \%$ (triangle), and $25 \%$ (circle). Tunnel capacitances and resistances are $C_{t}=0.1 \mathrm{fF}$ and $R_{t}=$ $300 \mathrm{k} \Omega$ pump/turnstile device ("h-pump") with two external drives. These devices should pass exactly one electron across the circuit per cycle of a periodic signal. Operational errors may occur due to missed cycles when frequency is too high, thermal activation, and cotunneling.

In Figure 2 we compare the current deviation from the quantized level $e f$ in those devices at the inflection points of their dc $I$ - $V$ curves as a function of temperature at $f=10 \mathrm{MHz}$. Because the main source of error in both pump and h-pump under zero bias is a cotunneling current in the opposite direction to the classical current [4], we simulated their operation using nonzero bias (a zero-biased pump is also displayed for comparison). "Robustness bars" show the current deviation if the inflection point is missed by some amount $(5,10$, and $25 \%$ of the correct bias point). Notice that the biased pump is more accurate than the non-biased pump by at least one order of magnitude. Notice also that, while the 8-junction performs poorly, the h-pump provide an accuracy comparable to that of the 5-junction pump.

\section{CONCLUSION}

We have developed SENECA, a new program for the calculation of probabilities of rare events in singleelectron systems, capable of analysis of circuits with arbitrary topology and parameters. It can find states with probabilities as small as desired, against the background of a large number of states with larger probabilities. The usefulness of the program has been demonstrated by a numerical studies of a single-electron trap and of the accuracy of several possible standards of dc current.

\section{Acknowledgements}

The work was supported in part by AFOSR and ONR/ ARPA. One of the authors (L. F.) was partially supported by CNPq-Brazil.

\section{References}

[1] Single Charge Tunneling, edited by H. Grabert and M. H. Devoret (Plenum Press, New York, 1992). 
[2] D. V. Averin and K. K. Likharev, in Single Charge Tunneling, edited by $\mathrm{H}$. Grabert and M. H. Devoret (Plenum Press, New York, 1992), p. 311.

[3] L. R. C. Fonseca, A. N. Korotkov, K. K. Likharev, and A. A. Odintsov, "A numerical study of the dynamics and statistics of single electron systems", J. Appl. Phys. 78, 3238 (1995).

[4] L. R. C. Fonseca, A. N. Korotkov, and K. K. Likharev, "A numerical study of the accuracy of single-electron current standards", in preparation.

\section{Biographies}

Leonardo R. C. Fonseca is a Ph.D. student in the Dept. of Applied Mathematics at Stony Brook. His research interests include software development, par- allel computing, numerical analysis, and simulation of mesoscopic phenomena.

Alexander N. Korotkov is a Research Scientist in the Dept. of Physics at Stony Brook. His research interests include physics and applications of singleelectron tunneling, and transport properties of semiconductor heterostructures.

Konstantin K. Likharev is a Professor of Physics of the State University of New York at Stony Brook. $\mathrm{He}$ has been active in the fields of nonlinear classical dynamics, quantum electronics, superconductor electronics, and low-temperature solid-state physics. 

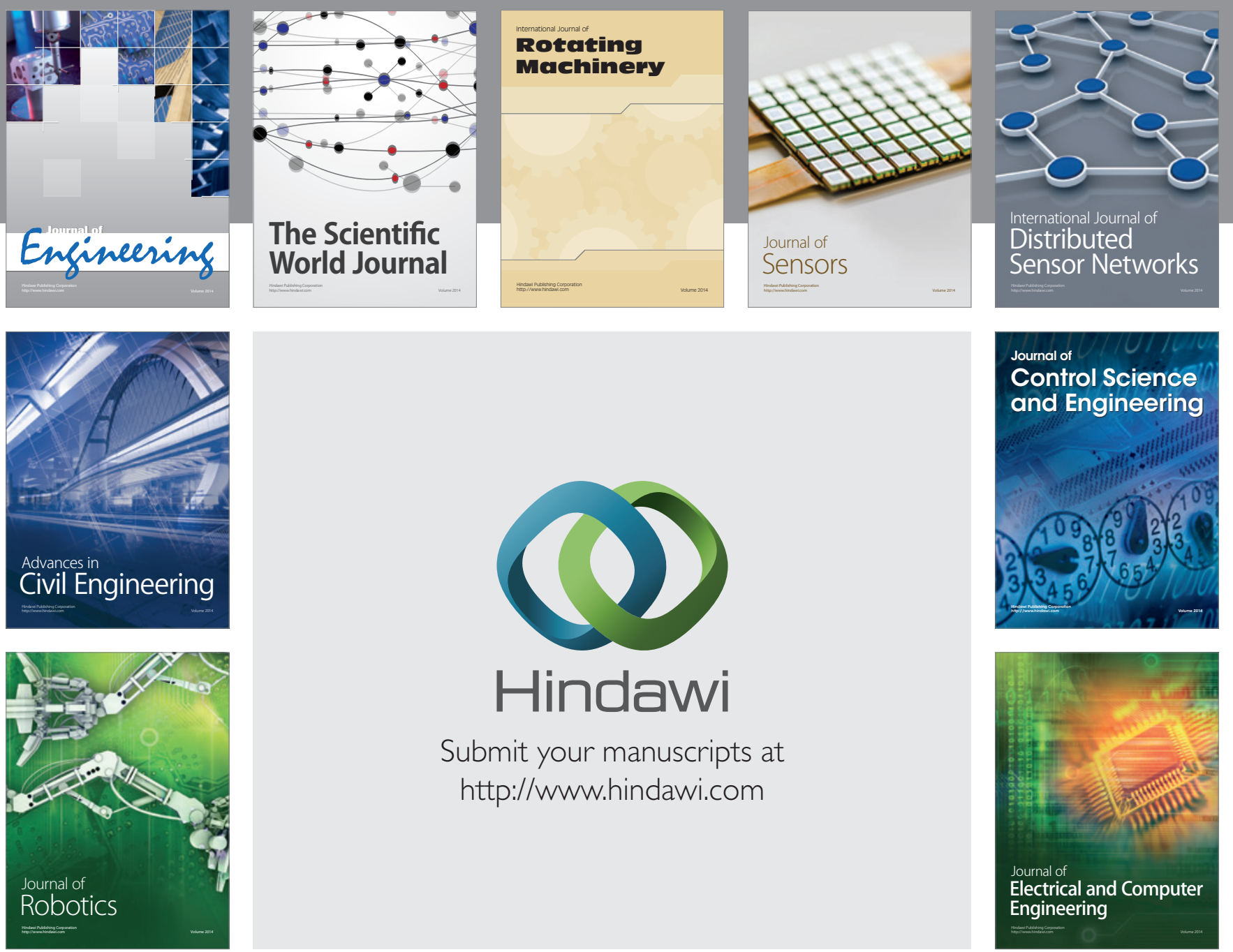

Submit your manuscripts at

http://www.hindawi.com
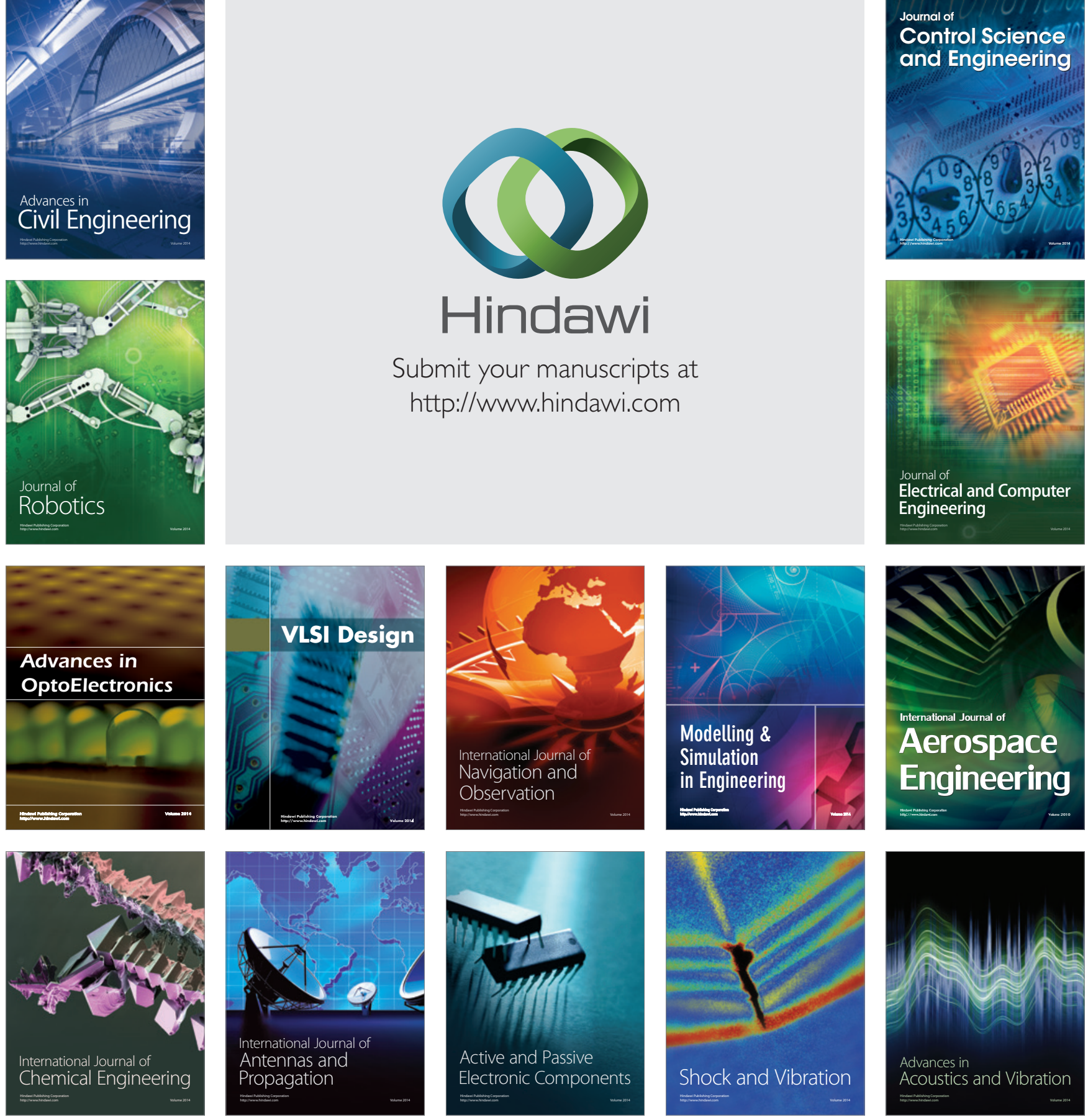